\title{
Multi-User Performance of the Amplify-and-Forward Single-Relay Assisted SC-FDMA Uplink
}

\author{
Jiayi Zhang, Lie-Liang Yang and Lajos Hanzo \\ School of ECS, University of Southampton, SO17 1BJ, UK. \\ Email: \{jz07r, lly, lh\}@ecs.soton.ac.uk; http://www-mobile.ecs.soton.ac.uk
}

\begin{abstract}
This paper characterises two single-relay assisted singlecarrier frequency-division multiple-access (SC-FDMA) amplify-andforward (AF) based cooperative strategies employed for multiuser uplink transmissions over multipath fading channels. For the sake of exploiting the benefits of cooperative diversity, we investigate both single-dedicatedrelaying (SDR) as well as single-shared-relaying (SSR), and propose a frequency-domain (FD) subband-based AF scheme combined with subband remapping, which is benchmarked against conventional timedomain (TD) AF in the context of both of the above-mentioned relaying topologies. Our simulation results show that cooperative diversity is beneficial for both SDR and SSR, both in the absence and in the presence of multipath diversity. More importantly, the proposed FD-AF scheme is capable of attaining a multiuser performance, which is better than that of the conventional single-user TD-AF protocol operating in a multipath environment, because of the subband-based $\mathrm{AF}$ and noise suppression capability of the relay.
\end{abstract}

\section{INTRODUCTION}

Wireless cooperative communications and relaying have attracted substantial research attention over the past decade as a benefit of their spatial diversity gain attained in a virtual multiple-input multiple-output (V-MIMO) scenario created by the distributed singleantenna terminals by sharing resources with their cooperative partners. This cooperation allows the system to mitigate the radio channel's fading by jointly exploiting the benefits of frequency, time- and spatial-diversity. Furthermore, the cooperative strategy may also be used to increase the attainable system capacity [1]-[4]. Recently, the diversity-multiplexing trade-off (DMT) encountered in multiple-access amplify-and-forward (MAF) relaying was considered for transmission over block fading channels in [5]. Moreover, both the single-user and multiuser performance of relay-assisted singlecarrier direct-sequence CDMA (SC DS-CDMA) [6] was discussed in [7], [8]. The relay-based single-carrier modulated frequencydomain equalisation (SC-FDE) [9], [10] aided space-frequency block coding (SFBC) assisted interleave-division multiple-access (IDMA) uplink (UL) was investigated in [11]. Prior studies considered relayaided cooperation both in a single-user and in a multiuser context, but their employment was typically restricted to transmission over frequency-flat fading channels. Naturally, at high bit rates the channel becomes frequency-selective and hence its deleterious effects have to be mitigated. Furthermore, both the multiuser interference (MUI) and inter-symbol-interference (ISI) should be cancelled. The associated processing imposes a high complexity, especially when the number of users is high.

Against this background, in this paper we investigate the cooperative diversity benefits achievable in the single-relay assisted single-carrier frequency-division multiple-access (SC-FDMA) uplink for transmission over frequency-selective fading channels. The SCFDMA technique advocated adopts SC-FDE combined with orthogo-

\footnotetext{
${ }^{1}$ The work reported in this paper has formed part of the Core 4 Research Programme of the Virtual Center of Excellence in Mobile and Personal Communications, Mobile VCE, www.mobilevce.com, whose funding support, including that of EPSRC, is gratefully acknowledged. Fully detailed technical reports on this research are available to Industrial Members of Mobile VCE.
}

nal subcarrier mapping in order to support multiple users without contamination by MUI. Furthermore, our single-relay assisted SC-FDMA system allows user cooperation to achieve a diversity gain without imposing MUI by the cooperating sources and relays upon the ULreceiver at the basestation (BS). It is worth noting that SC-FDMA is capable of exploiting the benefits of multipath diversity for transmission over broadband channels, while in orthogonal frequency-division multiplexing (OFDM) [12] each subcarrier experiences a single-path channel, hence no multipath diversity gain is achieved. It has been indicated in [13] that SC-FDMA attains a similar overall performance as orthogonal frequency division multiple access (OFDMA), but it is more suitable for uplink transmission due to its lower peak-toaverage power ratio (PAPR), which is typically high in multicarrier systems [12]. Additionally, the combination of based modulation with the discrete Fourier transform and inverse discrete Fourier transform (DFT/IDFT) SC-FDMA transmitter can be adaptively reconfigured in order to mitigate the effects of time-variant channels. The system is also available to cooperative communications. Hence, our novel contribution is that we achieve cooperative diversity by utilising a sophisticated SC-FDMA scheme, which exploits the benefits of AF based single-relay assisted user cooperation aided UL transmissions. Our simulation results show that cooperative diversity may be achieved for both the single-dedicated-relaying (SDR) and singleshared-relaying (SSR) strategies in both single-path and multipath scenarios. As a result, the proposed frequency-domain (FD) subbandbased amplify-and-forward (AF) scheme is combined with subband remapping at the relay. By contrast, the system invokes minimum mean-square error (MMSE) assisted FDE for each of the cooperative branches at the BS, where a simple time-domain (TD) equal-gain combiner (EGC) [14] is adopted. The resultant system is capable of achieving a multiuser performance, which is better than that of the conventional single-user TD-AF protocol operating in a multipath environment.

This treatise is organized as follows. In Section II, the system model of SC-FDMA is detailed, including both direct transmission and relay channels. In Section III, we propose the FD-AF aided cooperation with subband remapping, which will be compared to conventional TD-AF relaying in the context of both SDR and SSR schemes. We discuss our simulation results in Section IV and conclude in Section V.

\section{SYSTEM MODEL}

In the SC-FDMA system we investigated, $K$ mobile users are actived as source terminals, while the inactive nodes act as relays. The AF-based relay stations (RS) simply amplify the signal of each source without changing their carrier frequency. For the sake of separating multiple users in the FD and hence to avoid MUI, each source only selects a single relay node, which can be dedicated to a single user or may be shared by multiple users, based on the specific relaying topology shown in Fig. 1 according to the aforementioned SDR or SSR philosophy. Furthermore, we assume time division duplexing 


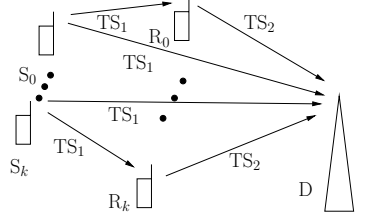

(a) Single-Dedicated-Relaying

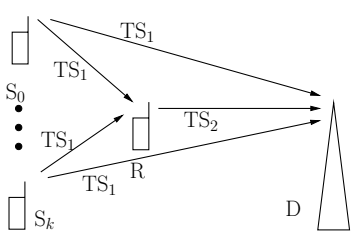

(b) Single-Shared-Relaying
Fig. 1. Relaying Topologies for Uplink Transmissions

(TDD) in the perfectly synchronised system considered. Observe in Fig. 1 that during the first time slot $\left(\mathrm{TS}_{1}\right)$, all the $K$ sources transmit their messages $\boldsymbol{s}_{S, k},(k=0,1, \cdots, K-1)$, which are simultaneously received by the RS(s) and by the BS via the source-to-relay (S-R) and the source-to-destination (S-D) link, respectively. Then, during the second time slot $\left(\mathrm{TS}_{2}\right)$, the RS forwards its received signal to the BS via the relay-to-destination (R-D) link according to the specific cooperative strategies to be described in Section III. We note that the effects of path-loss and shadowing have not been taken into account in this contribution, which implies the assumption of perfect power control for all links. The effects of realistic power control errors (PEC) will be considered in our future work. Furthermore, the total signal power of each user received via both the direct and relay branches was set to unity according to $P_{S, k}+P_{R, k}=1$.

\section{A. Transmitted Source Signal}

The SC-FDMA technique used in our work adopts the interleaved subband mapping aided DFT spread OFDM (DFT-S-OFDM) arrangement of [13], [15] at the transmitter. Specifically, the $N$-point DFT is used to transform the $k$-th user's $N$ consecutive time-domain (TD) symbols hosted by the vector $\boldsymbol{x}_{k}^{(t)}=\left[x_{k, 0}, x_{k, 1}, \cdots, x_{k, N-1}\right]^{T}$ of duration to $T_{v}$ into the FD, yielding $\boldsymbol{x}_{k}^{(f)}=\mathcal{F}_{N} \boldsymbol{x}_{k}^{(t)}$, which is constituted by $N$ orthogonal narrow subbands, where $\mathcal{F}_{N}$ denotes normalised $N$-point fast Fourier transform (FFT) matrix. Then, at the FD interleaving, the resultant $N$ FD symbols are mapped to orthogonal FD subbands which obeying the bandwidth expansion factor $M$, yielding a total bandwidth of $U=N \times M$. For further details on the SC-FDMA technique please refer to [13], [15]. Suffice to say that the baseband equivalent discrete-time expression of the transmitted singal before inserting the cyclic-prefix $(\mathrm{CP})$ is given by [15]

$$
\boldsymbol{s}_{S, k}^{(t)}=\sqrt{P_{S}} \mathcal{F}_{U}^{H} \boldsymbol{\mathcal { P }}_{k} \mathcal{F}_{N} \boldsymbol{x}_{k}^{(t)},
$$

where the superscript ${ }^{(t)}$ refers to the TD signal and $\mathcal{F}_{U}$ denotes the normalised $U$-point fast Fourier transform (FFT) matrix, while $\mathcal{P}_{k}$ is the subcarrier-mapping matrix of the $k$-th user having a normalised transmit power of $P_{S}$. To elaborate a little further, the FD subband mapping scheme, which is also known as subcarrier mapping in multicarrier systems, has to be designed to achieve the maximum attainable frequency diversity in dispersive multipath fading channels according to [15]

$$
\mathcal{P}_{k, \text { un }}=\left\{\begin{array}{lc}
1, & \text { for } u=n M+k \\
0, & \text { otherwise }
\end{array}\right.
$$

where $\mathcal{P}_{k, u n}$ is the $(u, n)$-th entry of $\mathcal{P}_{k}$ and we have $u=$ $0,1, \cdots, U-1, n=0,1, \cdots, N-1$.

\section{B. The BS's Received Signal via Direct Branch}

We assume that the BS perfectly estimated the channel state information (CSI) of the S-D link. Then, during $\mathrm{TS}_{1}$ the BS directly receives all the $K$ users' source signal via the S-D link subject to multipath fading. After removing the $\mathrm{CP}$, the received signal may be formulated as:

$$
\boldsymbol{r}_{S D}^{(t)}=\sum_{k=0}^{K-1} \tilde{\boldsymbol{H}}_{S D, k}^{(t)} \boldsymbol{s}_{S, k}^{(t)}+\tilde{\boldsymbol{n}}_{D 1}^{(t)}
$$

where the superscript refers to the $U$ vector length or $U \times U$ matrix size in the TD and $\tilde{\boldsymbol{H}}_{S D, k}^{(t)}$ represents the channel's circulant TD coefficient matrix [15] for the S-D link of the $k$-th source user, while $\tilde{\boldsymbol{n}}_{D 0}^{(t)}$ is the complex-valued additive white Gaussian noise (AWGN) component with zero mean and a variance of $\sigma_{N}^{2}$, i.e. we have $\mathcal{C N}\left(0, \sigma_{N}^{2}\right)$ at the BS.

In order to detect each user's signal in the FD at a low complexity, the subband demapping technique of [15] is implemented, followed by a $U$-point DFT. The subband demapping matrix is capable of removing the MUI imposed by all other users, leading to a singleuser vector of $N$ symbols, since we have

$$
\mathcal{P}_{k^{\prime}}^{T} \mathcal{P}_{k}=\left\{\begin{array}{lc}
\boldsymbol{I}, & \text { for } k^{\prime}=k \\
\mathbf{0}, & \text { for } k^{\prime} \neq k
\end{array}\right.
$$

where the user index at the BS's receiver is $k^{\prime}=0,1, \cdots, K-1$. Thus, this FD single-user $N$-symbol vector can be equalised by a single-tap MMSE equaliser [15], in order to suppress the effects of ISI. For the $k^{\prime}$-th user, the FDE's output vector $\hat{\boldsymbol{y}}_{S D, k^{\prime}}^{(f)}$ can hence be expressed as

$$
\hat{\boldsymbol{y}}_{S D, k^{\prime}}^{(f)}=\sqrt{P_{S}} \boldsymbol{W}_{S D, k^{\prime}}^{H} \boldsymbol{H}_{S D, k^{\prime}}^{(f)} \boldsymbol{x}_{k^{\prime}}^{(f)}+\hat{\boldsymbol{n}}_{D 0}^{(f)},
$$

where the superscript ${ }^{(f)}$ refers to the FD and $\boldsymbol{H}_{S D, k^{\prime}}^{(f)}=$ $\mathcal{P}_{k^{\prime}}^{T} \mathcal{F}_{U} \boldsymbol{H}_{S D, k^{\prime}}^{(t)} \mathcal{F}_{U}^{H} \boldsymbol{P}_{k^{\prime}}$ is the diagonal FD channel transfer factor (FDCHTF) matrix [12], [15], while the $n$-th $(n=0,1, \cdots, N-1)$ element of the $(N \times N)$ diagonal weight matrix $\boldsymbol{W}_{S D, k^{\prime}}$ of the MMSE-FDE is given by [15]

$$
w_{S D, k^{\prime} n}=P_{S} h_{S D, k^{\prime} n}^{(f)} /\left(\left|h_{S D, k^{\prime} n}^{(f)}\right|^{2} P_{S}+\sigma_{N}^{2}\right) \text {. }
$$

Additionally, $\hat{\boldsymbol{n}}_{D 0}^{(f)}=\boldsymbol{W}_{S D, k^{\prime}}^{H} \boldsymbol{\mathcal { P }}_{k^{\prime}}^{T} \mathcal{F}_{U} \boldsymbol{n}_{D 0}^{(t)}$ is the $(N \times N)$ diagonal noise matrix after equalisation. The processing of the $k^{\prime}$-th user's signal $\hat{\boldsymbol{y}}_{R D, k^{\prime}}^{(f)}$ received at the BS during $\mathrm{TS}_{2}$ depends on the specific choice of the relaying scheme, but this relayed signal is further combined with the direct signal $\hat{\boldsymbol{y}}_{S D, k^{\prime}}^{(f)}$ at the TD by a simple EGC in order to achieve spatial diversity in a virtual MIMO fashion, yielding

$$
\hat{\boldsymbol{y}}_{k^{\prime}}^{(t)}=\mathcal{F}_{N}^{H} \hat{\boldsymbol{h}}_{S D, k^{\prime}}^{(f)}+\mathcal{F}_{N}^{H} \hat{\boldsymbol{y}}_{R D, k^{\prime}}^{(f)} .
$$

\section{Single-Dedicated-Relaying}

In this single-relaying aided topology, we assume that there are many inactive mobile terminals avaiable which can play the role of relays during the cooperation phase in a cell. Each of these inactive mobile terminals receives and forwards the strongest signal received from a nearby source user, provided that it has a sufficiently high channel quality between them, while the interference imposed by the other users' signals is ignored due to their high distance. Hence, there are a total of $K$ sources and $K$ relays in the system. The signals received by the $k$-th RS as well as by the BS during $\mathrm{TS}_{1}$ and $\mathrm{TS}_{2}$, respectively, are expressed as follows,

$$
\begin{aligned}
\boldsymbol{r}_{S R, k}^{(t)} & =\tilde{\boldsymbol{H}}_{S R, k}^{(t)} \boldsymbol{s}_{S, k}^{(t)}+\tilde{\boldsymbol{n}}_{R, k}^{(t)}, \\
\boldsymbol{r}_{R D}^{(t)} & =\sum_{k=0}^{K-1} \tilde{\boldsymbol{H}}_{R D, k}^{(t)} \boldsymbol{s}_{R, k}^{(t)}+\tilde{\boldsymbol{n}}_{D 1}^{(t)},
\end{aligned}
$$

where $\tilde{\boldsymbol{H}}_{S R, k}^{(t)}$ hosts the TD channel coefficients of the S-R link for the $k$-th source user's signal, while $\tilde{\boldsymbol{n}}_{R, k}^{(t)}$ represents $\mathcal{C N}\left(0, \sigma_{N}^{2}\right)$ at the $k$-th relay. 
Moreover, for each user, both the source and the relay transmit their signal at the same normalised power, i.e. we have $P_{S}=P_{R}$. Hence, the UL system contains $K \mathrm{~S}-\mathrm{R}, \mathrm{S}-\mathrm{D}$ as well as the R-D links respectively, and the signals received via both the $S-R$ link and the R-D link experience $L$ independently fading multipath components. Naturally, every source also has its direct line-of-sight (LOS) link to the BS, which is also subject to independant frequency-selective fading.

It is important to note that, the AWGN imposed by the RS should be also taken into account. While the transmissions of the $k$-th user are mapped to $U$ subbands, the noise imposed by the RS is spread among all subbands in the FD, but only across the $N$ subbands belonging to the $k$-th user. On the other hand, when considering all the $K$ relays, there are $K$ noise which may degrade the BS's receiver's performance during $\mathrm{TS}_{2}$ when $K$ is increased. Additionally, when taking into account the BS receiver's noise contribution during $\mathrm{TS}_{1}$ and $\mathrm{TS}_{2}$, a total of $K+2$ noise sources having an overall average power at $\sigma_{N}^{2}$ exist in the system. Thus, the total noise power imposed by all the $K$ relays may become the dominant source of performance degradation, unless of course the noise effects are eliminated by more complex decode-and-forward (DF) relaying.

\section{Single-Shared-Relaying}

In contrast to the single-dedicated-relaying, when the number of inactive mobile stations becomes insufficient, the so-called SSR scheme may be invoked. Specifically, we assume that the RS is capable of receiving and forwarding signals from a cluster of $K$ source users, so that these sources can share the RS, which simultanously forwards their signals to the BS. Therefore, the representation of the signals received at the RS and BS is given by

$$
\begin{aligned}
\boldsymbol{r}_{S R}^{(t)} & =\sum_{k=0}^{K-1} \tilde{\boldsymbol{H}}_{S R, k}^{(t)} \boldsymbol{s}_{S, k}^{(t)}+\tilde{\boldsymbol{n}}_{R}^{(t)}, \\
\boldsymbol{r}_{R D}^{(t)} & =\tilde{\boldsymbol{H}}_{R D}^{(t)} \boldsymbol{s}_{R}^{(t)}+\tilde{\boldsymbol{n}}_{D 1}^{(t)},
\end{aligned}
$$

where, each of the $K$ source signals embedded in the forwarded messages is still transmitted on the same subcarriers as their used for transmission from source transmitters. Thus, the uplink system contains $K$ pairs of S-R and S-D links, but only a single R-D link. This implies that the transmissions containing the $K$-user signal forwarded from the RS to the BS is affected by the same multipath channel and contaminated by a single noise source at the RS. In other words, the performance degradation imposed by the RS is not related to the number of users $K$, which is in contrast to the SDR scheme of Subsection II-C.

However, it is unrealistic to provide sufficient RS power for the simultaneous transmission of $K$ users' signals. A simple realistic solution is to limit the total power of all the $K$ users to unity, i.e. to $P_{R}=1$. Then the average transmit power of each user's message at the source node has to be set to $P_{S}=1-1 / K$.

\section{Cooperative Strategies}

\section{A. Conventional TD-AF}

The classic AF scheme is based on analogue amplification of the received signal at the relay by a factor corresponding to the transmitted and received signal powers at relay in the TD [3]. In the SC-FDMA system, we assume that the BS perfectly estimated both the S-D and R-D links. The multiuser source signals are processed at the BS as follows.
For the SDR topology, we have $\boldsymbol{s}_{R, k}^{(t)}=\beta_{k}^{(t)} \boldsymbol{r}_{S R, k}^{(t)}$, the TD ampltification factor of the $k$-th relay is expressed as [3]

$$
\beta_{k}^{(t)}=\sqrt{P_{R} /\left\{\operatorname{Tr}\left[\tilde{\boldsymbol{H}}_{S R, k}^{(t)}\left(\tilde{\boldsymbol{H}}_{S R, k}^{(t)}\right)^{H}\right] P_{S} / U+\sigma_{N}^{2}\right\}} .
$$

The BS receives the messages forwarded from all the $K$ relays and processes them according to

$$
\hat{\boldsymbol{y}}_{R D, k^{\prime}}^{(f)}=\sqrt{P_{S}} \beta_{k^{\prime}}^{(t)} \boldsymbol{W}_{S R D, k^{\prime}}^{H} \boldsymbol{H}_{S R D, k^{\prime}}^{(f)} \boldsymbol{x}_{k^{\prime}}^{(f)}+\hat{\boldsymbol{n}}_{D 1}^{(f)},
$$

where $\boldsymbol{H}_{S R D, k^{\prime}}^{(f)}=\mathcal{P}_{k^{\prime}}^{T} \mathcal{F}_{U} \tilde{\boldsymbol{H}}_{R D, k^{\prime}}^{(t)} \tilde{\boldsymbol{H}}_{S R, k^{\prime}}^{(t)} \mathcal{F}_{U}^{H} \mathcal{P}_{k^{\prime}}$ represents the $k^{\prime}$ th user's FDCHTF matrixs for the S-R-D link, while for the R-D link, we have $\boldsymbol{H}_{R D, k^{\prime}}^{(f)}=\mathcal{P}_{k^{\prime}}^{T} \mathcal{F}_{U} \tilde{\boldsymbol{H}}_{R D, k^{\prime}}^{(t)} \mathcal{F}_{U}^{H} \mathcal{P}_{k^{\prime}}$. The total noise encounted of the BS in the FD includes the equivalent relaying noise imposed by the $K$ AF relays and the noise directly contributed by the BS's receiver during $\mathrm{TS}_{2}$, which is formulated as:

$$
\hat{\boldsymbol{n}}_{D 1}^{(f)}=\boldsymbol{W}_{S R D, k^{\prime}}^{H} \mathcal{P}_{k^{\prime}}^{T} \mathcal{F}_{U}\left[\sum_{k=0}^{K-1} \beta_{k}^{(t)} \tilde{\boldsymbol{H}}_{R D, k}^{(t)} \tilde{\boldsymbol{n}}_{R, k}^{(t)}+\tilde{\boldsymbol{n}}_{D 1}^{(t)}\right] .
$$

Hence, the MMSE-FDE's weight corresponding to the $n$-th subband is given by

$$
w_{S R D, k^{\prime} n}=\beta_{k^{\prime}}^{(t)} \sqrt{P_{S}} h_{S R D, k^{\prime} n}^{(f)} /\left[\left(\beta_{k^{\prime}}^{(t)}\right)^{2}\left|h_{S R D, k^{\prime} n}^{(f)}\right|^{2} P_{S}+\mathcal{N}_{D 1, n}\right] \text {, }
$$

where $\mathcal{N}_{D 1}$ represents the normalised total power of the equivalent noise imposed by the $K$ AF relays and the noise directly contributed by the BS's receiver during $\mathrm{TS}_{2}$, as given by

$$
\mathcal{N}_{D 1, n}=\sum_{k=0}^{K-1}\left(\beta_{k}^{(t)}\right)^{2}\left|h_{R D, k n}^{(f)}\right|^{2} \sigma_{N}^{2}+\sigma_{N}^{2} .
$$

However, in contrast to the SDR technique characterised in Eq. 12, for the SSR method, we have $\boldsymbol{s}_{R}^{(t)}=\beta^{(t)} \boldsymbol{r}_{S R}^{(t)}$, where the amplification factor of the SSR normalises the sum of all the $K$ source signals according to

$$
\beta^{(t)}=\sqrt{P_{R} /\left\{\sum_{k=0}^{K-1} \operatorname{Tr}\left[\tilde{\boldsymbol{H}}_{S R, k}^{(t)}\left(\tilde{\boldsymbol{H}}_{S R, k}^{(t)}\right)^{H}\right] P_{S} / U+\sigma_{N}^{2}\right\}} .
$$

After subband demapping, both $\hat{\boldsymbol{y}}_{R D, k^{\prime}}^{(f)}$ and $w_{S R D, k^{\prime} n}$ have the same expressions in Eq. (13) and (15) while replacing $\beta_{k^{\prime}}^{(t)}$ by $\beta^{(t)}$. However, the total received noise component of $k^{\prime}$-th user at the BS contains only a single RS's noise contribution, plus that of the BS, yielding the FD expression

$$
\hat{\boldsymbol{n}}_{D 1}^{(f)}=\boldsymbol{W}_{S R D, k^{\prime}}^{H} \mathcal{P}_{k^{\prime}}^{T} \mathcal{F}_{U}\left(\beta^{(t)} \tilde{\boldsymbol{H}}_{R D}^{(t)} \tilde{\boldsymbol{n}}_{R}^{(t)}+\tilde{\boldsymbol{n}}_{D 1}^{(t)}\right) .
$$

In addition, $\mathcal{N}_{D 1, n}$ represents the normalised power of the total received noise component at the $n$-th subband of the $k^{\prime}$-th user at the BS, which contains only a single RS's noise contribution, plus that of the BS during $\mathrm{TS}_{2}$, yielding

$$
\mathcal{N}_{D 1, n}=\left(\beta^{(t)}\right)^{2}\left|h_{R D, k^{\prime} n}^{(f)}\right|^{2} \sigma_{N}^{2}+\sigma_{N}^{2} .
$$

\section{B. Subband-Based FD-AF with Subband Remapping}

As we mentioned in Subsection II-C, the total equivalent noise of the SDR topology contaminating the BS's input signal is a function of the number of relays in the TD-AF system. Moreover, since the SSR topology forwards the received signals of all users from the $\mathrm{RS}$ to the BS, this TD-AF is unable to amplify the signal for each user differently. Additionally, we note for both the SDR and SSR architectures that the TD-AF scheme's signals transmitted in each of the subbands are amplified by the same factor for each user. However, provided that both the S-R and R-D links were perfectly 
estimated, the RS process them individually, including the subband remapping and amplification of each subband, while the BS is capable of equalising the two-hop relay link in the FD by a simple single-tap equaliser. Therefore, the proposed FD-AF scheme aims for reducing the ISI and the noise of each user.

To elaborate a little furtter, here we propose subband-based FD-AF with subband remapping for an SDR system. The transmitted signal of the $k$-th relay during $\mathrm{TS}_{2}$ is expressed as

$$
\boldsymbol{s}_{R, k}^{(t)}=\sqrt{P_{S}} \mathcal{F}_{U}^{H} \mathcal{P}_{k} \boldsymbol{\beta}_{k}^{(f)} \boldsymbol{H}_{S R, k}^{(f)} \boldsymbol{x}_{k}^{(f)}+\overline{\boldsymbol{n}}_{R, k}^{(t)},
$$

where $\boldsymbol{\beta}_{k}^{(f)}, \boldsymbol{H}_{S R, k}^{(f)}$ are the $k$-th user's FD amplification factor and the FDCHTF, respectively, both of which are $N \times N$ diagonal matrices. The $n$-th entry of $\boldsymbol{\beta}_{k}^{(f)}$ is given by

$$
\beta_{k n}^{(f)}=\sqrt{P_{R} /\left[\left|h_{S R, k n}^{(f)}\right|^{2} P_{S}+\sigma_{N}^{2}\right]},
$$

which affects the $n$-th subband signal as an equalisation weight in the FD. It is important to note that the noise contribution of the $k$-th user's signal is affected at RS as follows:

$$
\overline{\boldsymbol{n}}_{R, k}^{(t)}=\mathcal{F}_{U}^{H} \boldsymbol{\mathcal { P }}_{k} \boldsymbol{\beta}_{k}^{(f)} \boldsymbol{\mathcal { P }}_{k}^{T} \mathcal{F}_{U} \tilde{\boldsymbol{n}}_{R, k}^{(t)}
$$

where the noise imposed at other user's subbands are removed. Then the FD processing of the $k^{\prime}$-th user's signal received at the BS during $\mathrm{TS}_{2}$ can be described as:

$$
\hat{\boldsymbol{y}}_{R D, k^{\prime}}^{(f)}=\sqrt{P_{S}} \boldsymbol{W}_{S R D, k^{\prime}}^{H} \boldsymbol{H}_{R D, k^{\prime}}^{(f)} \boldsymbol{\beta}_{k^{\prime}}^{(f)} \boldsymbol{H}_{S R, k^{\prime}}^{(f)} \boldsymbol{x}_{k^{\prime}}^{(f)}+\hat{\boldsymbol{n}}_{D 1}^{(f)},
$$

where the total received noise component of the $k^{\prime}$-th user at the BS includes the noise contribution imposed by the $k^{\prime}$-th RS after the FDE plus that of the BS, is expressed as

$$
\hat{\boldsymbol{n}}_{D 1}^{(f)}=\boldsymbol{W}_{S R D, k^{\prime}}^{H} \boldsymbol{\mathcal { P }}_{k^{\prime}}^{T} \mathcal{F}_{U}\left(\tilde{\boldsymbol{H}}_{R D, k^{\prime}}^{(t)} \overline{\boldsymbol{n}}_{R, k^{\prime}}^{(t)}+\tilde{\boldsymbol{n}}_{D 1}^{(t)}\right) .
$$

The $k^{\prime}$-th user's MMSE FDE weight shown in Eq. (23) for the $n$-th subband of the R-D link is given by

$$
w_{S R D, k^{\prime} n}=\frac{P_{S} \beta_{k^{\prime} n}^{(f)} h_{R D, k^{\prime} n}^{(f)} h_{S R, k^{\prime} n}^{(f)}}{P_{S}\left(\beta_{k^{\prime} n}^{(f)}\right)^{2}\left|h_{R D, k^{\prime} n}^{(f)}\right|^{2}\left|h_{S R, k^{\prime} n}^{(f)}\right|^{2}+\mathcal{N}_{D 1, n}},
$$

where $\mathcal{N}_{D 1, n}$ has the same expression as Eq. (19), in which $\beta^{(t)}$ should be replaced by $\beta_{k^{\prime} n}^{(f)}$.

Considering the SSR, when the subband-based FD-AF approach is implemented, the transmitted signal containing all the $K$ users' signals by the RS is formulated as

$$
\boldsymbol{s}_{R}^{(t)}=\sum_{k=0}^{K-1}\left(\sqrt{P_{S}} \mathcal{F}_{U}^{H} \mathcal{P}_{k} \boldsymbol{\beta}_{k}^{(f)} \boldsymbol{W}_{S R, k}^{H} \boldsymbol{H}_{S R, k}^{(f)} \boldsymbol{x}_{k}^{(f)}+\overline{\boldsymbol{n}}_{R, k}^{(t)}\right) .
$$

Note that each user's signal forwarded by this common RS is divided by the number of sources $K$ in order to obey the unity-power constraint mentioned in Subsection II-C, yielding

$$
\beta_{k n}^{(f)}=\sqrt{P_{R} /\left[K\left(\left|h_{S R, k n}^{(f)}\right|^{2} P_{S}+\sigma_{N}^{2}\right)\right]} .
$$

Owning to the subband-based FD-AF, the FD processing obeying Eq. (23) and (25) and carried out at the BS's receiver is as the same as for the SDR scenario.

\section{Simulation Results and Discussions}

In this section, we provide a range of simulation results to characterise the achievable bit-to-error ratio (BER) performance of the single-relay assisted SC-FDMA system based on four different cooperative approaches investigated in this contribution, namely of the SDR TD-AF, SDR FD-AF, SSR TD-AF and SSR FD-AF schemes. Our parameters are summarized in Table I.
TABLE I

SIMULATION PARAMETERS

\begin{tabular}{|l|l|}
\hline Modulation, Coding & BPSK, no Channel Coding \\
\hline S-D, S-R, R-D Channels & Frequency-Selective Rayleigh \\
\hline Subband mapping Scheme & Interleaved \\
\hline$N:$ Number of subbands per user & 4 \\
\hline$M:$ Bandwidth expansion factor & 16 \\
\hline$U:$ Total number of subbands & 64 \\
\hline$K:$ Number of source users & $1,2,4,8,16$ \\
\hline$L:$ Number of paths & $1,2,4$ \\
\hline SDR Transmit power & $P_{S}=P_{R}=0.5$ \\
\hline SSR Transmit power & $K=1: P_{S}=P_{R}=0.5$, \\
& $K>1: P_{S}=1-P_{R} / K, P_{R}=1$ \\
\hline
\end{tabular}

Firstly, Fig. 2 depicts the BER versus $E_{b} / N_{0}$ performance of the SDR TD-AF assisted SC-FDMA uplink for different number of users and paths, which is compared to a non-cooperative benchmarker, when we have $N=4, M=16, L=1$ and $L=4$. Observe in Fig. 2 that a cooperative diversity gain is achieved by the SDR TD-AF scheme for a single-user system communicating over single-path fading channels. By contrast, for a multipath scenario, cooperative diversity gains are only achieved at high signal-to-noise ratios (SNRs) for a single-user scenario. Moreover, as a consequence of the excessive noise imposed by the RSs of the $K$ users across all subbands, the cooperative diversity gains of SDR are eroded in a multiuser scenario, when $K$ is increased. Additionally, compared to the single-path scenario, the multipath diversity gain provided by frequency-selective fading is retained, regardless of the number of users. The maximum diversity gain was achieved for $L=4$ when we had $N=4$.

Secondly, Fig. 3 characterises both the single-user and full-load multiuser BER performance of the SDR FD-AF approach upon varying the number of multipath components for $L=1,2,4$, compared to the corresponding non-cooperative scenarios using $N=4, M=16$. On one hand, as stated in Subsection III-B, in the SDR FD-AF scenario only a single RS contaminated the signal by the AWGN. Thus, the multiuser performance of the SDR FD-AF approach is the same as that of the single-user case supported by a single relay, where we have $L=1$ and 4 . On the other hand, a cooperative diversity gain is attained also in a multiuser scenario, when communicating over a single-path fading channel, which can be preserved for transmission over multipath channels, provided that the SNR is sufficiently high. Since the FD-AF scheme involves both subband remapping and subband-based AF processing which has a similar effect to that of a FDE at the RS, plus the MMSE-FDE at the BS for the entire relay branch, both the noise imposed by the RS as well as the ISI of the multipath environment have been suppressed at the BS. Hence, the achievable performance becomes better than that of the corresponding single-user performance of the SDR TD-AF scheme, where the same amplification factor was applied to all the subbands.

Thirdly, the single-user and multiuser performance of the SSR TDAF strategy are illustrated in Fig. 4 for $N=4, M=16, L=4$. A cooperative diveristy gain of $3-6 \mathrm{~dB}$ is achieved upon varying the number of users within the system for transmission over single-path channel for $N=4, M=16, L=4$ at BER of $10^{-4}$. However, since the multipath diversity gain is high, when we have $N=4, M=$ $16, L=4$, the cooperative diversity aided performance is eroded by the interference infected by the dispersive channel.

Finally, the BER performance of varying the number of users supported by the SSR FD-AF approach are demonstrated in Fig. 5 for $N=4, M=16, L=4$. Compared to the corresponding SSR TD-AF protocol, the cooperative diveristy gain is increased by both the single-user and multiuser system employing the SSR FD-AF scheme, particularly in a multipath environment, where in addition to the beneficial fading mitigation effect of multipath diversity, the 


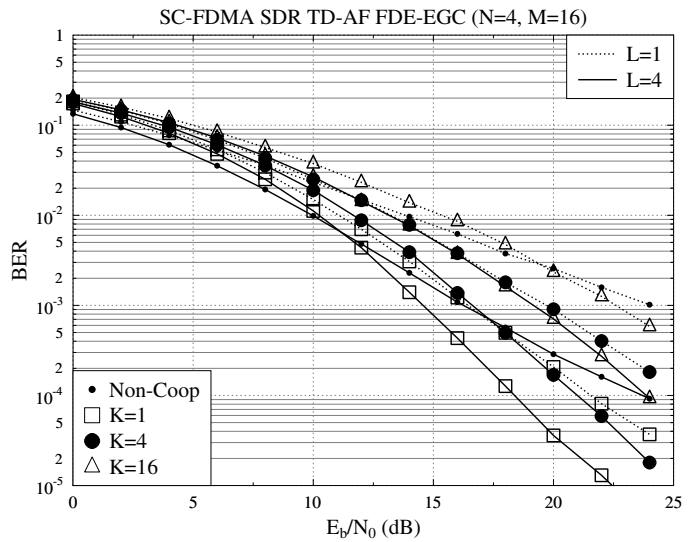

Fig. 2. BER versus $E_{b} / N_{0}$ performance of the SDR TD-AF scheme upon varying the number of users for $N=4, M=16, L=1,4$

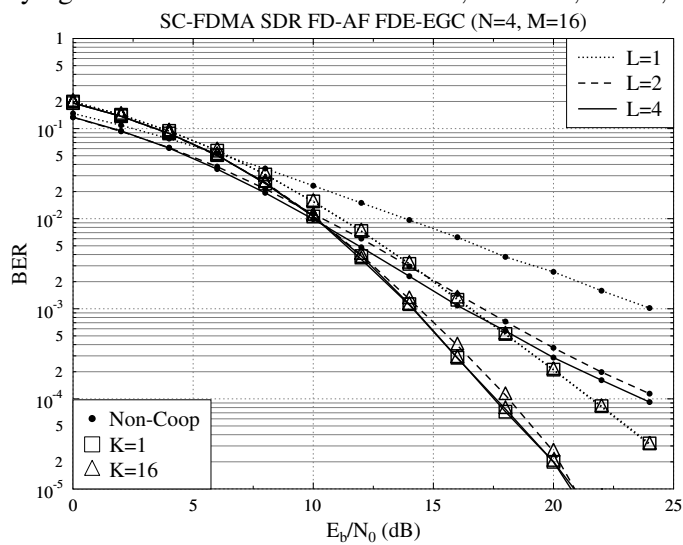

Fig. 3. BER versus $E_{b} / N_{0}$ performance of the SDR FD-AF scheme upon varying number of multipaths components for $N=4, M=16, K=16$

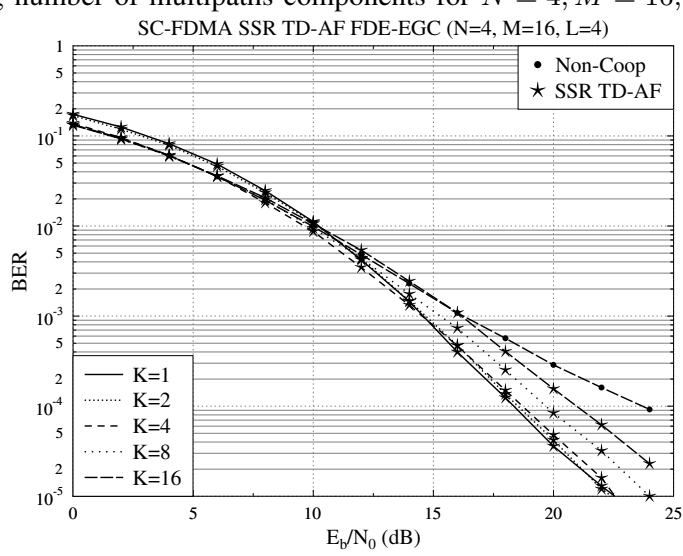

Fig. 4. BER versus $E_{b} / N_{0}$ performance of the SSR TD-AF scheme upon varying the number of users for $N=4, M=16, L=4$

interference imposed by the dispersive channel are also suppressed by including an subband-based AF at RS and the MMSE-FDE at the BS. The achievable cooperative diversity gain ranges from $5-8 \mathrm{~dB}$ for $N=4, M=16, L=4$ at a BER of $10^{-4}$ in the multiuser environment, as shown in Fig. 5.

\section{CONCLusions}

This paper investigated the performance benefits of subband-based FD-AF compared to the classic TD-AF based SDR and SSR topologies in the context of cooperative SC-FDMA uplink transmissions, which are free from any MUI at the RSs, when communicating over

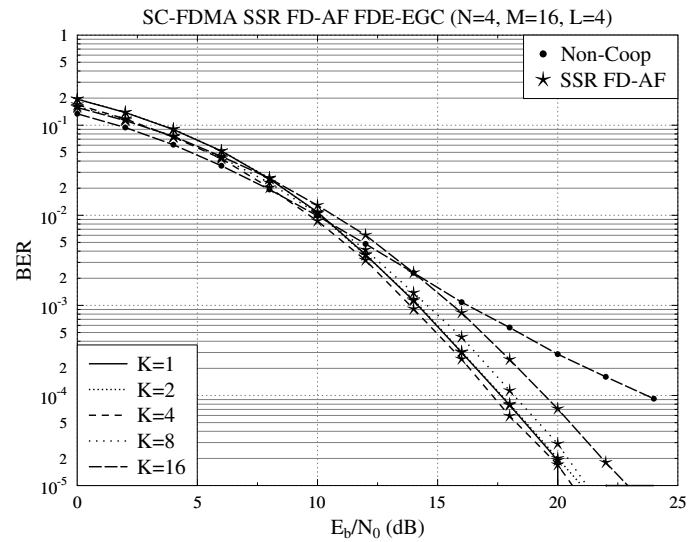

Fig. 5. BER versus $E_{b} / N_{0}$ performance of the SSR FD-AF scheme upon varying the number of users for $N=4, M=16, L=4$

frequency-selective fading channels. Our simulation results show that cooperative diversity may be achieved for both the SDR and SSR strategies in both single-path and multipath scenarios. As a result, the proposed subband-based FD-AF scheme is capable of achieving a multiuser performance, which is better than that of the conventional single-user TD-AF protocol operating in a multipath environment.

\section{REFERENCES}

[1] A. Sendonaris, E. Erkip, and B. Aazhang, "Increasing uplink capacity via user cooperation diversity," in Proceedings of IEEE International Symposium on Information Theory, p. 156, Aug. 1998.

[2] A. Sendonaris, E. Erkip, and B. Aazhang, "User cooperation diversity Part I and II," IEEE Transactions on Communications, vol. 51, pp. 19271948, Nov. 2003.

[3] J. N. Laneman, D. N. C. Tse, and G. W. Wornell, "Cooperative diversity in wireless networks: Efficient protocols and outage behavior," IEEE Transactions on Information Theory, vol. 50, pp. 3062-3080, Dec. 2004.

[4] R. U. Nabar, H. Bölcskei, and F. W. Kneubühler, "Fading relay channels: Performance limits and space-time signal design," IEEE Journal on Selected Areas in Communications, vol. 22, pp. 1099-1109, Aug. 2004.

[5] D. Chen, K. Azarian, and J. N. Laneman, "A case for amplify-forward relaying in the block-fading multiple-access channel," IEEE Transactions on Information Theory, vol. 54, pp. 3728-3733, Aug. 2008.

[6] L. Hanzo, L.-L. Yang, E.-L. Kuan, and K. Yen, Single- and Multi-Carrier DS-CDMA: Multi-User Detection, Space-Time Spreading, Synchronisation, Networking and Standards. Wiley, 2003.

[7] W. Fang, L.-L. Yang, and L. Hanzo, "Single-user performance of directsequence code-division multiple-access using relay diversity and power allocation," IET Communications, vol. 2, pp. 462-472, Mar. 2008.

[8] W. Fang, L.-L. Yang, and L. Hanzo, "Performance of relay-assisted DS-CDMA conflicting multiuser/inter-relay interference in nakagami$\mathrm{m}$ fading channels," in Proceedings of the IEEE Vehicular Technology Conference 2007 Fall, pp. 1027-1031, Sep., Oct. 2007.

[9] D. Falconer, S. L. Ariyavisitakul, A. Benyamin-Seeyar, and B. Eidson, "Frequency domain equalization for single-carrier broadband wireless systems," IEEE Communications Magazine, pp. 58-66, Apr. 2002.

[10] F. Pancaldi, G. M. Vitetta, R. Kalbasi, N. Al-Dhahir, M. Uysal, and H. Mheidat, "Single-carrier frequency domain equalization," IEEE Signal Processsing Magazine, pp. 37-56, Sept. 2008.

[11] T.-W. Yune, J.-B. Lim, Y.-C. Cheong, and G.-H. Im, "Iterative multiuser detection with spectral efficient protocol for relay-assisted SC-FDE," IEEE Communications Letters, vol. 12, pp. 182-184, Mar. 2008.

[12] L. Hanzo, M. Münster, B.-J. Choi, and T. Keller, OFDM and MC-CDMA for Broadband Multi-User Communications, WLANs and Broadcasting. Wiley, 2003.

[13] H. G. Myung, J. Lim, and D. J. Goodman, "Single carrier FDMA for uplink wireless transmission," IEEE Vehicular Technology Magazine, pp. 30-38, Sept. 2006.

[14] A. Goldsmith, Wireless Communications. Cambridge University Press, 2005.

[15] L.-L. Yang, Multicarrier Communications. Wiley, 2009. 